Miranda Morais, M., Burguera Condon, J.L., Arias Blanco, J.M. y Peña Suárez, E. (2019). Inclusión, diversidād y equidad: diseño y validación de un cuestionario de opinión dirigido al profesorado de orientación educativa (IDEC-0). Revista de Investigación Educativa, 37(2), 505-524.

DOI: http://dx.doi.org/10.6018/rie.37.2.333891

\title{
Inclusión, diversidad y equidad: diseño y validación de un cuestionario de opinión dirigido al profesorado de orientación educativa (IDEC-O)
}

\section{Inclusion, diversity and equity: design and validation of on opinion questionnarie adressed to school guidance counsellors (IDEC-O)}

\author{
Mirian Miranda Morais*, Joaquín Lorenzo Burguera Condon*, José Miguel Arias Blanco* \\ y Elsa Peña Suárez** \\ *Área de Métodos de Investigación y Diagnóstico en Educación. Facultad de Formación del Profesorado y Educación. \\ Universidad de Oviedo (España) \\ ***Consejería de Educación y Cultura. Principado de Asturias. (España)
}

\begin{abstract}
Resumen
El propósito de este trabajo es presentar el estudio Delphi realizado para diseñar y validar el contenido de un cuestionario de opinión elaborado ad hoc para recoger la opinión de orientadores y orientadoras sobre cómo los centros asturianos responden a la diversidad en educación primaria y secundaria. Se partió de un instrumento elaborado ad hoc del que se realizó una aplicación piloto en la que participaron 51 orientadores y orientadoras de secundaria $(N=51)$. Con objeto de adecuar la validez de contenido a ambas etapas se realizó un estudio Delphi de carácter cuantitativo y cualitativo en la que participaron 9 expertos. El estudio constó de dos rondas y distintas tareas con objeto de someter al criterio de expertos los bloques que componen el cuestionario y que recogen información sobre variables personales, profesionales, datos del centro, en una escala Likert de 48 items y preguntas abiertas. Se realizó un análisis de contenido de la información cualitativa y un análisis descriptivo, cálculo de la media, desviación típica y el porcentaje de acuerdo respecto a los criterios adoptados para considerar un ítem como válido. El resultado es un cuestionario de cinco bloques: tres recogen información sobre variables de identificación y clasificación (datos sociodemográficos; datos profesionales; datos referidos al
\end{abstract}

Correspondencia: Mirian Miranda Morais, miriandamirian@uniovi.es C/ Aniceto Sela, 1, 33005 Oviedo, Asturias. 
centro). El cuarto es una escala Likert de 45 items y cinco alternativas de respuesta (1= muy en desacuerdo hasta 5= muy de acuerdo). El último consta de tres preguntas abiertas sobre barreras, facilitadores y propuestas de mejora.

Palabras clave: orientadores escolares; orientación educativa; inclusión; atención a la diversidad; equidad.

\begin{abstract}
The aim of this paper focuses on the results of a Delphi study carried out to design and to validate the content of an "ad hoc" opinion questionnaire. The questionnaire has been developed to know the counselors' opinion on how the Asturian centers attend to diversity in primary and secondary stages. It was based on an instrument previously carried out, which was submitted to a pilot test in which 51 secondary school counselors participated $(N=51)$. In order to adapt the validity of the content to the two stages (primary and secondary), a quantitative and qualitative Delphi study was carried out in which 9 experts participated. The study was developed in two rounds and through different tasks in order to submit the blocks in which the questionnaire is structured to expert judgment. These blocks collect information on personal and professional variables and center data, on a Likert scale of 48 items and open questions. A content analysis of the qualitative information was carried out and a descriptive analysis of the mean, standard deviation and percentage of agreement in relation to the criteria adopted to consider an item as valid. The result is a questionnaire composed of five blocks: three of them collect information on identification and classification variables (sociodemographic, professional and center data). The fourth block is a Likert scale of 45 items and five response alternatives ( 1 = strongly disagree to 5 = strongly agree). The last one is formed by three open questions about barriers, facilitators and improvement proposals.
\end{abstract}

Keywords: educational counseling; inclusion; cultural differences; equity.

\title{
Introducción
}

La necesidad de una educación inclusiva efectiva está relacionada con el derecho a la educación y su interrelación con otros derechos humanos recogidos en tratados internacionales. Un hito importante en ese camino, es el impulso iniciado a partir de los años 90 con en la iniciativa "Educación para todos", así como los logros en la reclamación de los derechos para las personas con discapacidad entre los que cabe reseñar la Declaración de Salamanca (1994) y la Convención internacional sobre derechos de las personas con discapacidad $^{1}$ (Organización de las Naciones Unidas [ONU], 1994, 2006), cuyo artículo 24, referido a la educación, pone el acento en la necesidad de hacer efectivo este derecho sin discriminación sobre la base de la igualdad de oportunidades garantizando un sistema de educación inclusivo que se hace extensible a la totalidad del alumnado. A pesar del camino recorrido la Organización de las Naciones Unidas para la Educación, la Ciencia y la Cultura (UNESCO, 2015a, 2015b), que pone de relieve los desafíos por cumplir, y pone el acento en la educación concebida como un bien público y común, un derecho humano fundamental y la base para garantizar la realización de otros derechos.

\footnotetext{
1 Ratificada por el estado español en el año 2008 (BOE, número 96 de 21 de abril de 2008).
} 
El sistema educativo en España, regulado por la Ley Orgánica 2/2006, de 3 de mayo, de Educación (LOE, Boletín Oficial del Estado [BOE], de 4 de mayo de 2006) modificada por la Ley Orgánica 8/2013, de 9 de diciembre, para la Mejora de la Calidad Educativa (LOMCE, BOE de 10 de diciembre de 2013), refrenda estos derechos al reconocer la equidad, la inclusión, y la atención a la diversidad como principios fundamentales que deben regir la enseñanza para proporcionar a todo el alumnado una educación ajustada a sus características y necesidades, especialmente a aquellos más vulnerables.

A pesar de los avances realizados en política educativa, se observa una notable falta de sincronía respecto a lo que acontece en la práctica (e.g. Echeita, 2013; Echeita \& Verdugo, 2005; Simón \& Echeita, 2013). En concreto, se encuentran cuestiones mejorables relativas a la concepción y organización de los centros educativos; de la enseñanza; la actitud y formación del profesorado; la respuesta a la diversidad en la práctica docente; y la efectividad de las medidas (Arnáiz, 2009; Arnáiz \& Azorín, 2014; Arnáiz, Martínez, De Haro \& Escarbajal, 2013; Domínguez \& López, 2010; Ferrandis, Grau \& Fortes, 2010; Moliner, Sales, Traver \& Fernández, 2008; Rodríguez, 2013; Vázquez \& López, 2017). Esto supone, como plantea Bartolomé (2017), la necesidad de abordar nuevos enfoques que supongan un cambio en la concepción de la educación en general y en el sistema escolar en particular.

\section{Antecedentes y fundamentación teórica}

Este artículo se centra en el diseño y validación de un cuestionario dirigido a orientadores y orientadoras para conocer su opinión acerca de cómo los centros educativos asturianos que imparten la enseñanza obligatoria reconocen y garantizan la respuesta a la diversidad en base a los principios de inclusión y equidad.

En el Principado de Asturias no existe un desarrollo legislativo específico en materia de atención a la diversidad. Las referencias fundamentales son el Decreto 82/2014, de 28 de agosto, por el que se regula la ordenación y establece el currículo de la Educación Primaria en el Principado de Asturias (Boletín Oficial del Principado de Asturias [BOPA], de 30 de agosto de 2014) y el Decreto 43/2015, de 10 de junio, por el que se regula la ordenación y se establece el currículo de la Educación Secundaria Obligatoria en el Principado de Asturias (BOPA de 30 de junio de 2015). Estos dos decretos desarrollan en el Principado de Asturias lo establecido en la legislación de ámbito estatal. En ambos se señala que la atención a la diversidad tenderá a que todo el alumnado alcance los objetivos establecidos con carácter general y se regirá por los principios de "...calidad, equidad e igualdad de oportunidades, normalización, integración e inclusión escolar, igualdad entre mujeres y hombres, no discriminación, flexibilidad, accesibilidad universal y cooperación de la comunidad educativa" (BOPA, 2014, 2015; p.8).

Con objeto de contextualizar el cuestionario se partió de la definición que se recoge en la normativa, se entiende por atención a la diversidad "la orientación de la práctica educativa a dar respuesta a las diferentes capacidades, ritmos y estilos de aprendizaje, motivaciones e intereses, situaciones sociales, culturales, lingüísticas y de salud del alumnado" (artículo 20.1 del Decreto 82/2014, de 28 de agosto y artículo 16.1 del Decreto 43/2015, de 10 de junio). Asimismo, se parte de la concepción de la LOMCE (2013) al entender la orientación educativa y profesional como un medio necesario 
para el logro de una formación personalizada, que propicie una educación integral en conocimientos, destrezas y valores de todo el alumnado, y el papel que desempeñan los servicios especializados de orientación educativa como "...mecanismos creados para promocionar la equidad, es decir, ayudar en la tarea compleja de alcanzar los objetivos propios del éxito para todos, a través de prácticas pedagógicas caracterizadas por la inclusión y la justicia social" (Sánchez-Santamaría \& Ballester, 2014, p. 94)

A la hora de plantear la investigación y construir el instrumento se han tenido en cuenta la conceptualización y dimensiones de análisis del Index for Inclusion (Booth \& Ainscow, 2011; Booth, Simón, Sandoval, Muñoz, \& Echeita, 2015); el análisis de instrumentos sobre atención a la diversidad (Azorín, 2017; Azorín, Arnáiz, \& Maquilón, 2017); y la revisión de estudios dirigidos a conocer la opinión, actitud y percepción del profesorado sobre educación inclusiva, formación y práctica docente, respuesta a la diversidad, valoración de las medidas (e.g. Arnáiz, 2009; Arnáiz \& Azorín, 2014; Arnáiz et al., 2013; Ferrandis et al., 2010; Moliner et al. 2008; Rodríguez, 2013). Y especialmente los estudios desarrollados por Domínguez y López (2010) y Vázquez y López (2017) destinados a recoger la opinión de los orientadores sobre la atención a la diversidad en educación primaria y secundaria, respectivamente.

El diseño de un instrumento dirigido específicamente al profesorado de orientación educativa como agente informante viene justificado por una doble vía. La primera es que se trata de los únicos agentes en los centros educativos que cuentan con una formación inicial y de acceso al cuerpo docente específica en esta materia. La segunda viene determinada por el rol que desempeñan en el centro, ya que entre sus competencias profesionales (Vélaz de Medrano, Manzanares Moya, López Martín, \& Manzano Soto, 2013) y sus funciones, recogidas en el contexto de esta investigación en el Decreto 147/2014, de 23 de diciembre, por el que se regula la orientación educativa y profesional en el Principado de Asturias ${ }^{2}$ (BOPA, de 29 de diciembre de 2014), se encuentra la responsabilidad de colaborar de manera activa en la planificación, seguimiento, implementación y evaluación de la respuesta a la diversidad en los centros basada en los principios de inclusión y equidad educativa.

La necesidad y valor añadido de diseñar un instrumento dirigido a este colectivo radica en la importancia de recoger la voz de profesionales del ámbito escolar superando una de las limitaciones expuestas por Azorín (2017) respecto a que la mayor parte de la investigación en esta materia proviene del ámbito universitario.

\section{Diseño y propiedades del cuestionario inicial.}

Para la construcción del "IDEC-O" se partió de un instrumento elaborado ad hoc por el equipo de investigación (Miranda, 2013; Miranda, Burguera, \& Arias, 2015). Se realizó una exhaustiva revisión bibliográfica y se tuvo en cuenta la normativa en materia de atención a la diversidad y orientación educativa a nivel estatal y autonómico. A partir de ese análisis, se construyó un banco de ítems siguiendo las últimas propuestas y recomendaciones emitidas por las instituciones internacionales expertas

2 Para ampliar información sobre el modelo, estructura y funciones de la Orientación Educativa y Profesional en el Principado de Asturias consultar el Decreto. 
en la materia (i.e. American Educational Research Association [AERA], American Psychological Association [APA], \& National Council on Measurements in Education [NCME, 2014]; Haladyna, Downing, \& Rodríguez, 2002; Moreno, Martínez, \& Muñiz, 2006; Muñiz \& Fonseca-Pedrero, 2008).

El proceso se inició con un banco de 62 ítems que fueron sometidos al juicio de expertos para la validación de contenido ${ }^{3}$. Como resultado se obtuvo una escala Likert de 55 ítems de 5 opciones de respuesta (de 1=Muy en desacuerdo a 5=Muy de acuerdo). El equipo investigador incorporó en el cuestionario varias preguntas para recoger información de variables sociodemográficas y profesionales de los informantes y tres preguntas abiertas para identificar aspectos que favorecen y dificultan la respuesta a la diversidad en los centros de secundaria y formular propuestas de mejora. Posteriormente, se realizó una prueba piloto en la que participaron 51 orientadores y orientadoras de educación secundaria, que suponen un $63.75 \%$ del total de la población en Asturias ( $\mathrm{N}=80$ ). El grado de consistencia de la escala (con los 55 ítems) se analizó mediante el estadístico alpha de Cronbach obteniéndose un valor de 0.91. Posteriormente se llevó a cabo un análisis de discriminación y consistencia interna de los ítems manteniendo en la escala aquellos que mostraban una discriminación igual o superior a 0.20 . Como resultado se obtuvo una escala final de 48 ítems con un valor del alpha de Cronbach de 0.91. Tomando como referencia de interpretación el baremo que establece Nunnally (1978) se trata de un valor excelente. También se llevó a cabo un Análisis Factorial Exploratorio (AFE), mediante el método de máxima verosimilitud, en el que se obtuvieron 11 factores con autovalores mayores que 1 pero con un primer factor dominante que explica el $31.407 \%$ de la varianza total. La estructura del cuestionario que se ha tomado como punto de partida para la elaboración del IDEC-O se presenta en la Tabla 1.

Tabla 1

Estructura del cuestionario inicial "La opinión del Profesorado de Orientación Educativa de la atención a la diversidad en la ESO"

\begin{tabular}{lll}
\hline Bloques & \multicolumn{1}{c}{ Variables } & \multicolumn{1}{c}{ Tipo de preguntas } \\
\hline Bloque I & Sociodemográficas y de clasificación & $\begin{array}{l}\text { Items y preguntas de diferentes } \\
\text { formas (opciones, elección, abier- } \\
\text { tos) }\end{array}$ \\
Bloque II & $\begin{array}{l}\text { Atención a la diversidad en Educación } \\
\text { Secundaria Obligatoria }\end{array}$ & $\begin{array}{l}\text { Escala Likert 48 ítems con 5 op- } \\
\text { ciones (1=Muy en desacuerdo, a } \\
5=\text { Muy de acuerdo) }\end{array}$ \\
Bloque III & $\begin{array}{l}\text { Aspectos que favorecen y dificultan la } \\
\text { respuesta a la diversidad. Propuestas de } \\
\text { mejora }\end{array}$ & Preguntas abiertas \\
\hline
\end{tabular}

3 El grupo de trabajo estaba formado por 10 personas y se constituyó en el curso 2013-14. 


\section{Método}

\section{Objetivos}

Los objetivos que se han planteado para este trabajo son:

- Diseñar un cuestionario dirigido al profesorado de orientación educativa (IDEC-O) que permita recoger su opinión acerca de cómo los centros que imparten educación primaria y secundaria responden a la diversidad atendiendo a cuestiones relacionadas con la cultura, política de centro, práctica docente, formación y actitud del profesorado, y evaluación de las actuaciones.

- Estudiar la validez de contenido del cuestionario IDEC-O a través de un estudio Delphi.

\section{Participantes}

A fin de obtener la validación de contenido se contó con la colaboración del profesorado que forma parte de una actividad de innovación y formación formalizada (Grupo de Trabajo Intercentros de Orientación Educativa ${ }^{4}$ ). En el momento que se solicitó su participación en el panel Delphi el grupo estaba formado por 15 orientadores y orientadoras con perfiles heterogéneos en cuanto a características profesionales (experiencia en orientación, antigüedad en el centro, etapa educativa y experiencia laboral previa) y personales (edad, género y formación previa).

Con objeto de garantizar la validez de contenido del cuestionario y la generalización al conjunto de la población se fijaron los siguientes criterios de inclusión: formar parte del grupo de trabajo y haber trabajado en servicios especializados de orientación de centros que impartan la educación primaria y/o secundaria. A la hora de seleccionar los participantes en el estudio Delphi se estimó pertinente incluir a personas con perfiles heterogéneos respecto a variables profesionales "etapa educativa en la que prestan servicios" y "años de experiencia en servicios de orientación". Se consideró relevante equilibrar la participación en el proceso de validación del profesorado en función de la etapa educativa, primaria y secundaria, para evitar sesgos y asegurar que el contenido de las preguntas e ítems de la escala se adecuasen a ambas etapas. En el caso de contar con una experiencia menor a los 5 años como orientador/a, se tuvo en cuenta para su inclusión que contasen con una trayectoria académica y profesional previa destacable.

Finalmente, un $73.33 \%(\mathrm{~N}=11)$ de la población cumplía los criterios de inclusión y mostró interés por colaborar. Con el fin de equilibrar la participación en función de la etapa educativa en la que prestan servicios (primaria o secundaria) se descartó a dos

4 Esta actividad de formación se enmarca en la convocatoria de innovación y formación permanente del profesorado del Principado de Asturias. Se trata de una actividad formativa referente en Asturias del Centro de Profesorado y Recursos de Avilés-Occidente con una trayectoria de más de 10 años. Su finalidad se concreta en proporcionar un espacio de reflexión sobre la acción, establecer redes de apoyo entre profesionales y crear conocimiento compartido. En la actualidad, curso 2017-18, está constituido por 36 orientadores y orientadoras. Este grupo se ha comprometido con la investigación desde sus inicios y por ello se solicitó su colaboración en este proceso de validación. 
profesionales de secundaria. Finalmente, el grupo invitado al estudio Delphi quedó compuesto por 9 participantes.

\section{Procedimiento}

El estudio Delphi se desarrolló de acuerdo a las siguientes fases atendiendo a las consideraciones expuestas en el trabajo realizado por López-Gómez (2018):

\section{Fase I. Profundización en la revisión bibliografía. Análisis de la normativa sobre atención a la diversidad y orientación educativa en el Principado de Asturias.}

Ante la necesidad de que las preguntas e ítems que componen el instrumento se adecuasen a las características de educación primaria y secundaria, se realizó una revisión normativa y bibliográfica que abordase la respuesta a la diversidad en el conjunto de la enseñanza básica. Atendiendo a estas consideraciones se optó por realizar las siguientes modificaciones en la estructura del cuestionario:

1. Variables sociodemográficas y profesionales. Se añadió el tipo de servicio especializado de orientación que corresponde a primaria: Unidades de Orientación y Equipos de Orientación Educativa (ver Apéndice 1. Bloque II del cuestionario IDEC-O).

2. Variables relativas al centro educativo. Se incluyó la tipología (público y privado concertado), tamaño, población, alumnado con necesidades específicas de apoyo educativo, recursos de apoyo especializado y participación del centro en programas de innovación y formación (ver Apéndice 1. Bloque III del cuestionario IDEC-O).

3. Escala Likert. Se revisó la adecuación del contenido de los 48 ítems iniciales a la etapa de primaria. Se concluyó que estos ítems eran transferibles, por lo que se decidió no descartar ninguno y someter los 48 ítems al juicio de expertos.

Además, se realizó una revisión conceptual de las dimensiones en las que se agrupan los ítems de la escala. Para ello se tomaron como referencia las dimensiones recogidas en la Guía para la Educación Inclusiva: desarrollando el aprendizaje y la participación en los centros escolares (Booth et al., 2015), versión adaptada para el uso en centros educativos españoles y países iberoamericanos del Index for Inclusion: developing learning (Booth \& Ainscow, 2011), al tratarse de un enfoque contrastado a nivel internacional que tiene en consideración los planos fundamentales de la vida escolar de cualquier centro educativo en las etapas de infantil, primaria y secundaria. Las dimensiones del IDEC-O se encuentran en línea con las propuestas por estos autores: "Cultura de centro" (relaciones, valores y creencias arraigadas en la comunidad educativa); "Políticas de centro" (gestión del centro y planificación e implementación de planes y programas) y "Práctica docente" (refleja aspectos relativos a las prácticas del aula). También, de acuerdo con la literatura e investigaciones citadas, se añade una dimensión específica "Formación docente" que recoge aspectos relativos a la actitud y formación, al tratarse de dos aspectos clave en el desarrollo e investigación de los procesos inclusivos (Azorín, 2017; Azorín et al, 2017). Por último, se incluye la dimensión "Evaluación" referida al seguimiento de las medidas 
de atención a la diversidad, su impacto en la mejora de resultados y éxito académico del alumnado, dada la importancia que se le concede a la evaluación basada en evidencias como elemento de mejora del sistema educativo desde el ámbito académico y la legislación educativa. En la Tabla 2 se recogen las dimensiones y descriptores en los que se reorganizaron y agruparon los ítems que conforman la escala.

Tabla 2

Dimensiones y descriptores en los que se agrupan los ítems que conforman la escala Likert.

\begin{tabular}{|c|c|}
\hline Dimensiones & Descriptores \\
\hline \multirow[t]{2}{*}{ Cultura de centro } & Valores y creencias \\
\hline & Comunidad y participación \\
\hline \multirow[t]{3}{*}{ Política de centro } & Gestión de centro \\
\hline & Organización y funcionamiento \\
\hline & Planes y programas \\
\hline \multirow[t]{2}{*}{ Práctica docente } & Concreción curricular \\
\hline & Procesos de enseñanza - aprendizaje \\
\hline \multirow{2}{*}{$\begin{array}{l}\text { Formación do- } \\
\text { cente }\end{array}$} & Legislación educativa \\
\hline & Formación inicial y continua \\
\hline \multirow[t]{2}{*}{ Evaluación } & Seguimiento \\
\hline & Resultados \\
\hline
\end{tabular}

En cuanto al bloque formado por preguntas abiertas, el equipo de investigación optó por mantenerlo sin modificaciones al considerar su pertinencia y adecuación para identificar condiciones, procesos y prácticas que favorecen y dificultan la respuesta a la diversidad; e indagar en la formulación de propuestas de mejora que favorezcan el desarrollo de procesos inclusivos desde la labor cotidiana de los centros educativos de ambas etapas.

Finalizado este proceso se elaboró un primer borrador que consta de una estructura de cinco bloques que incluyen preguntas e ítems de diversa tipología y opciones de respuestas para someterlo al proceso de validación utilizando el método Delphi.

\section{Fase II. Estudio Delphi: diseño y planificación.}

Se llevó a cabo un estudio Delphi desde una perspectiva cuantitativa y cualitativa, para validar el contenido de las preguntas e ítems que componen el IDEC-O. El equipo investigador estableció los criterios de inclusión para participar en el panel Delphi y las 
condiciones éticas, se trasladó la información a la población invitada, y se diseñaron las tareas y el procedimiento de recogida de información y análisis de datos.

\section{Fase III: Procedimiento de recogida de información}

Se mantuvo una primera reunión con las 9 personas invitadas que voluntariamente aceptaron participar para informarles sobre los aspectos básicos de la investigación (tipo de estudio, objetivos y aspectos éticos). Para garantizar la confidencialidad y anonimato de las respuestas se solicitó que se asignaran un pseudónimo. Las tareas fueron elaboradas mediante formularios on-line y se enviaron a través de correo electrónico acompañadas de todo el material necesario (instrucciones, cuestionario y tareas). El procedimiento de recogida de información se desarrolló en dos rondas:

\section{Ronda I}

Consistió en cuatro tareas: (1) contestar un formulario referido a variables sociodemográficas y profesionales; (2) valorar los 48 ítems que conforman la propuesta inicial de la escala en función de dos criterios: pertinencia (evidencias acerca de si el ítem evalúa la respuesta a la diversidad en la enseñanza básica) y relevancia (evidencias acerca de si el ítem resulta importante para conocer cómo se aplica o desarrolla la atención a la diversidad en los centros) con una escala de 1 a 4; (3) proponer nuevos ítems y (4) realizar sugerencias a las preguntas, ítems y opciones de respuesta de los bloques del cuestionario dirigidos a recoger información de variables sociodemográficas, profesionales y de centro.

\section{Ronda II}

Finalizada la primera ronda, se analizaron los datos y los resultados fueron sometidos a la segunda ronda del estudio Delphi. Constó de dos tareas: (1) valorar los ítems que en la primera ronda no habían superado el corte fijado respecto a uno de los dos criterios establecidos (pertinencia o relevancia), atendiendo a las siguientes alternativas: $\mathrm{SI}=$ mantenerlo en el cuestionario; $\mathrm{NO}=$ suprimirlo y $\mathrm{R}=$ reformularlo; (2) valorar la pertinencia y relevancia de los ítems reformulados y nuevos obtenidos como resultado de la ronda I utilizando el procedimiento descrito en la tarea 2 de la Ronda I.

\section{Fase IV: Análisis de los datos}

Para lograr los objetivos planteados y analizar los datos obtenidos en el estudio Delphi se han empleado los siguientes métodos. Respecto a la información cualitativa se ha realizado un análisis de contenido de las sugerencias planteadas por las personas expertas. Para el análisis cuantitativo de datos obtenidos de las tareas de valoración de la escala se ha utilizado el programa IBM SPSS para Windows (versión 20). Se ha calculado en la primera ronda la valoración de los ítems en cuanto a su pertinencia y relevancia respecto a la escala (de 1 a 4). Se realizó un análisis de las medias de cada uno de los ítems en cuanto a pertinencia y relevancia. El criterio adoptado para con- 
siderar los ítems como válidos fue el siguiente: 1) presentar una media superior a 3 y una desviación típica inferior o igual a 1.5; y 2) presentar valoraciones de 3 o 4 en, al menos, el $80 \%$ de las respuestas. Se siguió el mismo criterio en la segunda ronda para valorar los ítems nuevos $(\mathrm{N}=4)$ sugeridos por los expertos en la primera ronda.

\section{Resultados}

\section{Ronda I}

\section{Resultados Tarea I. Características sociodemográficas y profesionales}

La media de edad de los 9 participantes se sitúa en los 45 años y el 33.33\% (N=3) son hombres. Todo ellos cuentan, al menos, con el título de Licenciatura (Pedagogía, Psicología o Psicopedagogía). Un 22.22\% (N=2) cursó previamente Magisterio y una participante es Doctora en Psicología con experiencia de investigación. El 66.66\% $(\mathrm{N}=6)$ cuenta con amplia experiencia como profesorado de Orientación, entre los 10 y los 27 años en estas etapas educativas y la totalidad tiene una trayectoria profesional previa en el ámbito socioeducativo. El 44.44\% (N=4) trabaja en educación secundaria, otro $44.44 \%(\mathrm{~N}=4)$ en primaria y una persona en un CPEB que imparte ambas etapas. El 88.88\% ( $\mathrm{N}=8)$ ocupa puestos de coordinación de la Unidad o la Jefatura del Departamento de Orientación.

\section{Resultados Tarea 2. Valoración de los ítems de la escala Likert}

Los análisis de concordancia mostraron que 33 ítems (68.75\%) cumplían con todos los criterios de validez establecidos. El criterio de pertinencia se cumplió para el 83.33\% de los ítems $(\mathrm{N}=40)$, el de relevancia en el $77.08 \%$ de los ítems $(\mathrm{N}=37)$ y hubo 4 ítems $(8.33 \%)$ que no superaron los valores establecidos en ambos criterios y, en consecuencia, fueron eliminados. Los 11 ítems ( $\mathrm{N}=22.92 \%)$ que incumplían uno de los criterios (pertinencia o relevancia) fueron sometidos al foro de discusión de la segunda ronda.

Finalmente, como resultado de la primera ronda se mantiene un $91.66 \%(\mathrm{~N}=44)$ de los ítems del banco inicial, de los que un $68.75 \%(n=33)$ pasa a formar parte de la escala IDEC-O al cumplir los criterios de validación establecidos en esta primera ronda.

\section{Resultados Tarea 3. Proponer nuevos ítems}

Los expertos propusieron un total de 4 ítems nuevos, que fueron valorados en la segunda ronda. Previamente, se evaluó el cumplimiento de recomendaciones de directrices internacionales sobre la redacción de ítems por el equipo investigador.

\section{Resultados Tarea 4. Valorar preguntas, ítems y opciones de respuesta}

Como resultado del análisis de contenido de las aportaciones realizadas se obtuvieron, en mayor término, alternativas de modificación de la redacción para mejorar la comprensión. Cabe señalar, la práctica ausencia de propuestas respecto al contenido 
de los ítems, preguntas y opciones planteadas. En el Apéndice adjunto al instrumento se presenta la versión final (Bloques I, II y III) exceptuando la parte de la escala Likert (Bloque IV) y preguntas abiertas (Bloque V).

\section{Ronda II}

\section{Resultados Tarea I.Valoración de los ítems que no cumplan algún criterio de pertinencia o relevancia}

En la Ronda II contamos con 11 ítems sometidos a la opinión de los jueces (N=9). A partir de los resultados, expuestos en la Tabla 3, se decide no eliminar ningún ítem al no ser rechazado por un porcentaje igual o superior al $44.44 \%(\mathrm{~N}=4)$, mantener 7 ítems aceptados por un porcentaje igual o superior al 77.78\% (N=7) y reformular 3 ítems que fueron propuestos por un porcentaje igual o mayor al $22.22 \%(\mathrm{~N}=2)$. Contamos con un ítem "Los equipos docentes discrepan sobre las medidas que se han de adoptar para dar una respuesta al alumnado" que cumplía requisitos para mantenerse y para ser reformulado. En este caso, analizado el contenido, se decide modificar su redacción por una de las sugerencias aportadas: "Los equipos docentes desarrollan las medidas que se deben adoptar para dar una respuesta individualizada al alumnado".

Tabla 3

Resultados de la Tarea 1. Ronda II. Ítems escala Likert ( $n=11)$.

\begin{tabular}{lcc}
\hline Respuestas & No de ítems seleccionados & \% de ítems seleccionados \\
\hline Mantener & 7 & 72.72 \\
Reformular & 4 & 36.36 \\
Eliminar & 0 & 0 \\
\hline
\end{tabular}

\section{Resultados Tarea 2. Valorar ítems nuevos propuestos en la Ronda I.}

El procedimiento fue el mismo que el utilizado en la tarea 2 de la Ronda I. Los participantes valoraron 4 ítems en pertinencia y relevancia, resultando 2 de ellos válidos para ser incluidos en la versión final de la escala.

Un ítem que había sido reformulado "El profesorado del alumnado con NEE carece de horario para coordinarse con los/as especialistas" fue sustituido por un ítem nuevo al tratarse del mismo contenido formulado en positivo "El profesorado del alumnado con NEE dispone de horario para coordinarse con los/as especialistas".

\section{Síntesis del panel Delphi}

La Figura 1 resume los principales resultados en cada una de las rondas y la composición final de la escala Likert. 


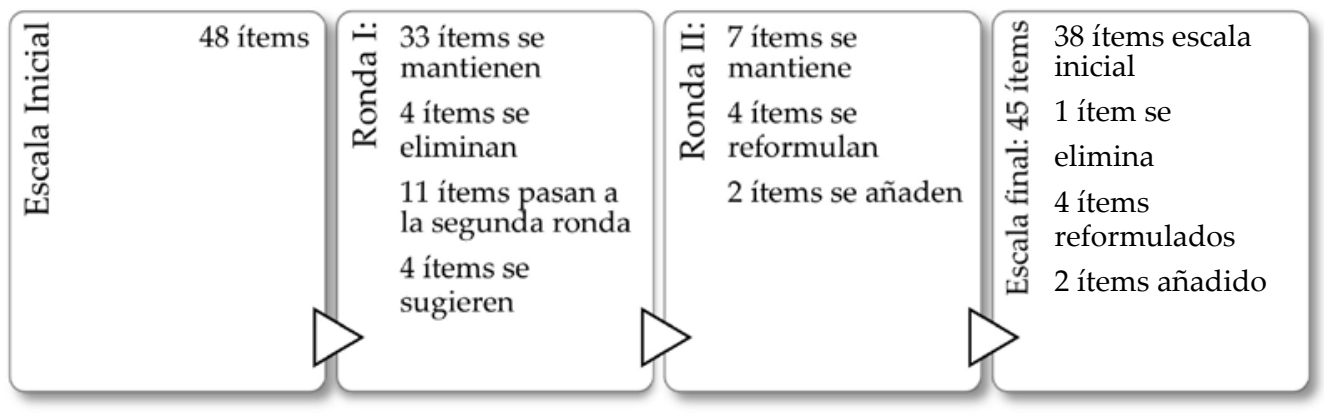

Figura 1. Resumen resultados del proceso de validación de la escala Likert.

\section{Versión final del IDEC-O}

Finalizado el estudio Delphi, el equipo de investigación se centró en la redacción de la versión final del cuestionario IDEC-O, ver apéndice 1, que responde a la estructura que se muestra en la Tabla 4:

Tabla 4.

Estructura del cuestionario.

\begin{tabular}{|c|c|c|}
\hline Bloque & Información & Tipo de preguntas \\
\hline $\mathrm{I}$ & $\begin{array}{l}\text { Variables sociodemográficas (edad, sexo y } \\
\text { formación) }\end{array}$ & \\
\hline II & $\begin{array}{l}\text { Variables profesionales (experiencia, antigüe- } \\
\text { dad en el puesto, formación y cargo) }\end{array}$ & $\begin{array}{l}\text { Preguntas e ítems de di- } \\
\text { versa tipología (abiertas, } \\
\text { de opciones...) }\end{array}$ \\
\hline III & $\begin{array}{l}\text { Variables centro (localidad, titularidad, tipo- } \\
\text { logía, enseñanzas, alumnado, recursos del } \\
\text { servicio de orientación, participación en pro- } \\
\text { gramas de innovación y formación) }\end{array}$ & \\
\hline IV & $\begin{array}{l}\text { Respuesta a la diversidad. } \\
\text { Facetas: cultura de centro ( } 11 \text { ítems), políti- } \\
\text { ca (14 ítems), práctica ( } 7 \text { ítems), formación ( } 5 \\
\text { ítems) y evaluación (8 ítems) }\end{array}$ & $\begin{array}{l}\text { Escala Likert } 45 \text { ítems de } \\
\text { cinco alternativas de res- } \\
\text { puesta ( } 1=\text { Muy en des- } \\
\text { acuerdo hasta } 5=\text { Muy de } \\
\text { acuerdo) }\end{array}$ \\
\hline $\mathrm{V}$ & $\begin{array}{l}\text { - Aspectos que facilitan y dificultan la aten- } \\
\text { ción a la diversidad. } \\
\text { - Propuestas de mejora de atención a la diver- } \\
\text { sidad centros. }\end{array}$ & Preguntas abiertas \\
\hline
\end{tabular}

Para facilitar el procedimiento de recogida de información se optó por construir tres tipos diferenciados de cuestionarios como se expone en la Figura 2. 


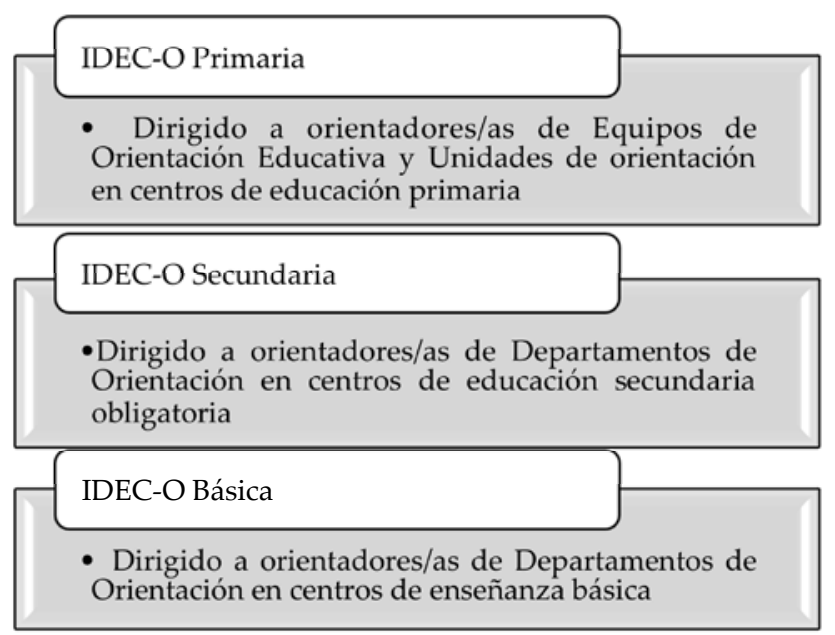

Figura 2. Versiones del IDEC-O adaptadas a las distintas etapas y tipologías de centros.

En el caso del IDEC-O B, se duplican las preguntas del Bloque III referidas a la etapa educativa (alumnado de la etapa, alumnado con NEE y ACNEE), y se duplica el Bloque IV, Escala Likert, en el que han de contestar a los 45 ítems que componen la escala respecto a la etapa de Primaria y a la etapa de Secundaria.

\section{Discusión y conclusiones}

A partir de los resultados obtenidos y teniendo en consideración los objetivos formulados presentamos la discusión de aquellos en relación a otros estudios sobre el tema, se enuncian conclusiones y se sugieren futuras líneas de actuación.

El primer objetivo planteado fue diseñar un cuestionario dirigido a orientadores/as que permita recoger información respecto a si los centros responden a los principios de atención a la diversidad, inclusión y equidad garantizando el derecho del alumnado a recibir una educación de calidad en las enseñanzas básicas. Para ello se partió de un instrumento que contaba con buenas propiedades psicométricas (Miranda et al. 2015) y se tomaron como referencia los avances recogidos en política educativa a nivel internacional sobre educación inclusiva (ONU, 2006; UNESCO, 2015a, UNESCO, 2015b), su conceptualización en el Index for inclusion (Booth \& Ainscow, 2011), la visión empírica aportada desde distintas investigaciones (Arnáiz, 2009; Arnáiz \& Azorín, 2014; Arnáiz et al., 2013; Domínguez \& López, 2010; Ferrandis et. al, 2010; Moliner et. al, 2008; Rodríguez, 2013; Vázquez \& López, 2017) y lo dispuesto en materia de inclusión educativa y atención a la diversidad a nivel estatal y autonómico. Dado que el objeto de estudio es la educación básica desde la visión de los servicios especializados de orientación, se tuvieron en cuenta las etapas educativas, las tipologías y características de los centros y los aspectos sociodemográficos y profesionales de la muestra. Esta revisión y el conocimiento de lo que acontece en los centros a través de la participación de los orientadores/as ha permitido ajustar el contenido a lo que sucede en la práctica. 
Como consecuencia, se modificó la estructura del instrumento de partida, añadiendo dos nuevos bloques, y se elaboró una nueva versión del instrumento (Miranda et al., 2015).

Respecto al segundo objetivo, centrado en estudiar la validez de contenido del cuestionario IDEC-O a las etapas de educación primaria y secundaria, se desarrolló un estudio Delphi en el que participaron 9 orientadores/as. Para garantizar el proceso, se atendió a los parámetros metodológicos señalados por López-Gómez (2018). En este panel de expertos se sometió a evaluación la totalidad de los ítems, preguntas, opciones de respuesta y escala Likert, a través de dos rondas. Los datos fueron exhaustivamente analizados desde la perspectiva cuantitativa y cualitativa y sus resultados conformaron la versión final del IDEC-O, del que se han diseñado tres versiones para facilitar su aplicación.

Como principal conclusión podemos señalar que el instrumento que se presenta ha sido sometido a un proceso riguroso que avala la validez de contenido-y responde a los objetivos propuestos. Por tanto, este estudio contribuye a profundizar en el constructo de atención a la diversidad, operativizando dicho constructo a través de la elaboración, adaptación y análisis del cuestionario IDEC-O a las etapas de educación primaria y secundaria.

Por otra parte, implicar de manera directa a los orientadores/as en el proceso de diseño del instrumento ha permitido obtener información contextualizada sobre las prácticas que acontecen en la realidad educativa; propiciando un proceso de reflexión sobre su práctica y lo que acontece a los centros, que les ha permitido adquirir experiencia y mejorar sus competencias profesionales en el ámbito de la investigación educativa (Vélaz de Medrano et al., 2013).

En cuanto a las limitaciones, al tratarse de un cuestionario dirigido a estos profesionales, no recoge la voz de todos los agentes que participan del escenario educativo (Azorín, 2017; Azorín et al., 2017). Asimismo, al haber contextualizado la investigación a la enseñanza básica en Asturias, se cuenta con una muestra y población limitada.

Como futuras líneas de investigación se plantea la utilización del IDEC-O como instrumento de autoevaluación de la respuesta a la diversidad en los centros, así como su combinación con otras técnicas que permitan ampliar las evidencias sobre los procesos educativos que contribuyen a la implementación de planes de innovación y mejora en consonancia con otras investigaciones como la realizada por Arnáiz y Azorín (2014).

Asimismo, se plantea la administración de dicho cuestionario a una muestra más amplia y representativa de la población de los profesionales de la orientación educativa en el Principado de Asturias, con objeto de profundizar en el conocimiento de las políticas inclusivas y medidas de atención a la diversidad que se aplican en esta comunidad y conocer el contexto profesional de los servicios de orientación educativa.

En línea con lo anteriormente comentado, y de acuerdo con lo que expone Azorín (2017), consideramos esencial dedicar esfuerzos y recursos a investigar y diseñar instrumentos de evaluación para esta materia que recojan la visión de otros colectivos que forman parte de la práctica educativa: alumnado, familias, personal no docente, asesores, inspección educativa, etc., que nos permitan conocer si se está garantizando el derecho de todo el alumnado a recibir una educación de calidad basada en los principios de inclusión, equidad y respuesta a la diversidad. 
Al contar con los profesionales que forman parte activa de la realidad educativa se pretende contribuir a abrir nuevas líneas de investigación en materia de atención a la diversidad y educación inclusiva que permitan avanzar y construir conocimiento de manera colaborativa recogiendo evidencias para definir líneas de actuación que contribuyan a hacer efectivo el derecho de todo el alumnado a recibir una educación de calidad con la finalidad de superar los retos expuestos por la UNESCO (2015a, 2015b).

\section{Referencias}

American Educational Research Association, American Psychological Association, \& National Council on Measurement in Educatión. (2014). Standards for Educational and Psychological Testing. Washington D.C.: Author.

Arnáiz, P. (2009). Análisis de las medidas de atención a la diversidad en la educación secundaria obligatoria. Revista de Educación (349), 203-223. Recuperado de http://www. revistaeducacion.mec.es/re349/re349_10.pdf

Arnáiz, P., \& Azorín Abellán, C. M. (2014). Autoevaluación docente para la mejora de los procesos educativos en escuelas que caminan hacia la inclusión. Revista Colombiana de Educación (67), 227-245. Recuperado de http://revistas.pedagogica.edu.co/index. php/RCE/article/view/2878/2586

Arnáiz, P., Martínez, R., De Haro, R., \& Escarbajal, A. (2013). Anlysis of measures for attention to diversity in compulsary secondary education: The case of the Region of Murcia, Spain. Journal of Research in Special Educational Needs (13), 189-197. Recuperado de https://www.um.es/documents/787838/1466270/analysis-measures-attentiondiversity_2011.pdf/ec7a3c24-ccde-432c-9d2a-26e2192b9e6f

Azorín, C. M. (2017). Análisis de instrumentos sobre educación inclusiva y atención a la diversidad. Revista Complutense de Educación, 28(4), 1043-1060. doi: http://dx.doi. org/10.5209/RCED.51343

Azorín, C. M., Arnáiz, P., \& Maquilón, J. J. (2017). Revisión de instrumentos sobre atención a la diversidad para una educación inclusiva de calidad. Revista mexicana de investigación educativa, 22(75), 1021-1045. Recuperado de https://bit.ly/2RXt234

Bartolomé, M. (2017). Diversidad educativa ¿Un potencial desconocido? Revista de Investigación Educativa, 35(1), 15-33. doi: http://dx.doi.org/10.6018/rie.35.1.275031

Booth, T., \& Ainscow, M. (2011). Index for Inclusion. Developing learning and participation in schools. Bristol: CSIE.

Booth, T., Simón, C., Sandoval, M., Muñoz, Y., \& Echeita, G. (2015). Guía para la educación inclusiva. Promoviendo el aprendizaje y la participación en los centros escolares. Nueva edición revisada y ampliada. Revista Iberoamericana sobre Calidad, Eficacia y Cambio en Educación, 13(3), 5-19. Recuperado de https://revistas.uam.es/index. php/reice/article/view/2780/2995

Decreto 82/2014, de 28 de agosto, por el que se regula la ordenación y establece el currículo de la Educación Primaria en el Principado de Asturias. Boletín Oficial del Principado de Asturias. Principado de Asturias, 30 de agosto de 2014, núm. 202, pp. 1-414.

Decreto 147/2014, de 23 de diciembre, por el que se regula la orientación educativa y profesional en el Principado de Asturias. Boletín Oficial del Principado de Asturias. Principado de Asturias, de 29 de diciembre de 2014, núm. 299, pp. 1-14. 
Decreto 43/2015, de 10 de junio, por el que se regula la ordenación y se establece el currículo de la Educación Secundaria Obligatoria en el Principado de Asturias. Boletín Oficial del Principado de Asturias. Principado de Asturias, de 30 de junio de 2015, núm. 150, pp. 1-521.

Domínguez, J., \& López, A. (2010). Funcionamiento de la atención a la diversidad en la enseñanza primaria según la percepción de los orientadores. Revista de Investigación en Educación, (7), 50-60. Recuperado de http://sid.usal.es/idocs/F8/ART15609 /funcionamiento_atencion_diversidad.pdf

Echeita, G. (2013). Inclusión y Exclusión Educativa. De nuevo "Voz y quebranto". Revista Iberoamericana sobre Calidad, Eficacia y Cambio en Educación, 11(2), 99-118. Recuperado de https://revistas.uam.es/index.php/reice/article/view/2899

Echeita, G., \& Verdugo, M. A. (2005). Diez años después de la Declaración de Salamanca sobre necesidades educativas especiales en España. Entre la retórica esperanzadora y las resistencias al cambio. SIGLO CERO. Revista Española sobre Discapacidad Intelectual, 36(1) (213), 5-12. Recuperado de http://riberdis.cedd.net/handle/11181/3107

Ferrandis, M. V., Grau, C., \& Fortes, M. C. (2010). El profesorado y la atención a la diversidad en la ESO. Revista de Educación Inclusiva, 3(2), 11-28. Recuperado de http:// riberdis.cedd.net/handle/11181/4650

Haladyna, T., Downing, S., \& Rodríguez, M. (2002). A review of multiple-choice itemwriting guidelines. Applied Measurement in Education, 15(3), 309-334. Recuperado de https://sites.educ.ualberta.ca/staff/ldelia/M'Choice'Tests= Haladyna_Guidelines_ AME_2002.pdf

Ley Orgánica de Educación 2/2006, de 3 de mayo. Boletín Oficial del Estado. España, núm. 106, de 4 de mayo de 2006, pp. 17158- 17207.

Ley Orgánica 8/2013, de 9 de diciembre, para la mejora de la calidad educativa. Boletín Oficial del Estado. España, núm. 295, de 10 de diciembre de 2013, pp. 97858- 97921.

López-Gómez, E. (2018). El método Delphi en la investigación actual en educación: una revisión teórica y metodológica. Educación XX1, 21(1), 17-40. doi: 10.5944/educXX1.15536

Miranda, M. (2013). La atención a la diversidad en la ESO: una mirada desde la orientación educativa (TFM). Recuperado de http://hdl.handle.net/10651/18346

Miranda, M., Burguera, J. L., \& Arias, J. M. (2015). La atención a la diversidad en los centros de secundaria: diseño y propiedades de un cuestionario de opinión dirigido a orientadores. En AIDIPE (Ed.), Investigar con y para la sociedad (Vol. 1, pp. 103-112). Cádiz: Asociación Interuniversitaria de investigación Pedagógica.

Moliner, O., Sales, A., Traver, J. A., \& Fernández, R. (2008). La atención a la diversidad en los centros de Educación Secundaria Obligatoria: análisis de las variables facilitadoras y limitadoras de las prácticas docentes. Educación y Diversidad (2), 99-127. Recuperado http://repositori.uji.es/xmlui/handle/10234/17693

Moreno, R., Martínez, R., \& Muñiz, J. (2006). New guidelines for developing multiplechoice items. Methodology: European Journal of Research Methods for the Behavioral and Social Sciences 2(2), 65-72. doi: http://dx.doi.org/10.1027/1614-2241.2.2.65

Muñiz, J., \& Fonseca-Pedrero, E. (2008). Construcción de instrumentos de medida para la evaluación universitaria. Revista de Investigación en Educación, 5, 13-25. Recuperado de http://reined.webs.uvigo.es/index.php/reined/article/view/41/30 
Nunnally, J. C. (1978). Psychometric theory. New York: McGraw-Hill

Organización de las Naciones Unidas (1994). Declaración de Salamanca y Marco de acción para las necesidades educativas especiales. Recuperado de https://bit.ly/1wiQz1c

Organización de las Naciones Unidas (diciembre, 2006). Convención de la ONU sobre los derechos de las personas con discapacidad. Nueva York, EE.UU.: Sede de las Naciones Unidas.

Organización de las Naciones Unidas para la Educación, la Ciencia y la Cultura (2015a). Replantear la Educación ¿Hacia un bien común mundial? Recuperado de https://bit. ly/2rC1sgc

Organización de las Naciones Unidas para la Educación, la Ciencia y la Cultura (2015b). Educación 2030. Declaración de Incheon y Marco de acción para la realización del objetivo de desarrollo sostenible 4. Garantizar una educación inclusiva, equitativa y de calidad y promover oportunidades de aprendizaje durante toda la vida para todos. Recuperado de https://bit. ly/2A1UmpS

Rodríguez, A. M. (2013). Modelos de Atención a la Diversidad en Educación Secundaria Obligatoria: Análisis Comparativo de los Planes de Atención a la Diversidad de las Comunidades Autónomas de Andalucía y de la Región de Murcia. Revista Nacional e Internacional de Educación Inclusiva, 6(3), 41-66. Recuperado de http://riberdis.cedd. net/bitstream/handle/11181/4235/modelos_atencion_diversidad_educacion_secundaria_obligatoria.pdf?sequence=1\&rd=0031359019024711

Sánchez-Santamaría, J., \& Ballester, M. G. (2014). Desarrollando el éxito educativo para todos: reflexiones, propuestas y retos conceptuales en torno a la calidad educativa. Revista Iberoamericana sobre Calidad, Eficacia y Cambio en Educación, 12(2), 85-104. Recuperado de https://revistas.uam.es/index.php/reice/article/view/2857/3074

Simón, C., \& Echeita, G. (2013). Comprender la educación inclusiva para intentar llevarla a la práctica. En H. Rodríguez \& L. Torrego (Coords.), Educación inclusiva, equidad y derecho a la diferencia. Transformando la escuela (pp. 33-65). Madrid: Wolters Kluver España.

Vázquez, E., \& López, E. (2017). El diagnóstico en la diversidad desde la perspectiva de la orientación en secundaria. Revista de Estudios e Investigación en Psicología y Educación, Vol.Extr.(11), 96-100. doi: https://doi.org/10.17979/reipe.2017.0.11.2444

Vélaz de Medrano, C., Manzanares, A., López-Martin, E., \& Manzano-Soto, N. (2013). Competencias y formación de los orientadores escolares. Estudio empírico en nueve Comunidades Autónomas. Revista de Educación, (nº Extr.), 261-292. doi: 10.4438/1988592X-RE-2013- EXT-249

Fecha de recepción: 7 de junio de 2018.

Fecha de revisión: 20 de junio de 2018.

Fecha de aceptación: 1 de abril de 2019. 


\title{
Apéndice I. Inclusión, Diversidad y Equidad. Cuestionario para orientadores y orientadoras (IDEC-O)
}

\author{
IDEC-O \\ Inclusión, Diversidad y Equidad: cuestionario para orientadores y orientadoras
}

El objetivo del presente cuestionario es recoger tu visión como Orientador/a sobre la respuesta a la atención a la diversidad en los centros educativos respecto a la Etapa de Educación Primaria y la Educación Secundaria Obligatoria. No te llevará más de 10 minutos cubrirlo. Consta de cuatro páginas: Primera. Datos sociodemográficos y profesionales; Segunda. Datos referidos al centro; Tercera. Escala Likert. Atención a la diversidad y Cuarta. Cuestiones sobre aspectos que favorecen y dificultan la atención a la diversidad.

Para pasar de una página a otra, debe pulsar en el botón CONTINUAR. Para que las respuestas queden registradas

Te agradecemos que respondas con sinceridad, según tu opinión profesional. El cuestionario es anónimo. No se trabajará con las respuestas individuales de cada persona sino con el conjunto de los datos obtenidos.

\begin{tabular}{|c|c|c|c|}
\hline \multicolumn{4}{|c|}{ BLOQUE I. DATOS SOCIO-DEMOGRÁFICOS } \\
\hline $\begin{aligned} & \text { Sexo: } \\
& \square \\
& \square\end{aligned}$ & $\begin{array}{l}\text { Hombre } \\
\text { Mujer }\end{array}$ & Edad: & 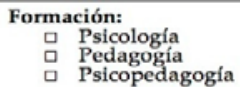 \\
\hline
\end{tabular}

\section{BLOQUE II. DATOS PROFESIONALES}

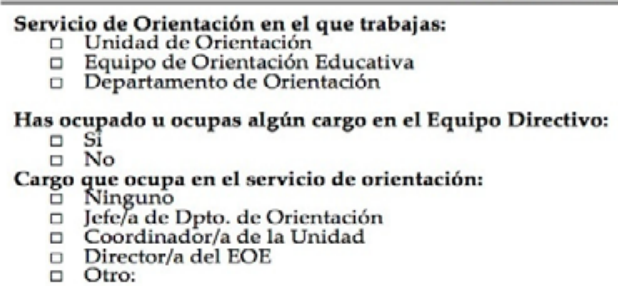
Años de experiencia como orientador:
마 1 a 5
ㅁ de 6 a 10
$\square$ de 11 a 15
ㅁ de 16 a 20
a de 21 a 25
más de 25

Años de experiencia en el centro:
ㅁ de 1 a 5
ㅁ de 6 a 10
ㅁ de 11 a 15
ㅁ de 16 a 20
ㅁ de 21 a 25

\section{BLOQUE III. CENTRO}

A continuación se recogen algunas cuestionas referidas al centro educativo en el que trabajas o en el caso de pertenecer a un EOE debes elegir un centro, en el que se imparta la etapa de primaria y en el que tengas asignada mayor presencia horaria, para aportar los datos referidos al mismo.

Localidad:
Tipo:
$\square$ CP
$\square$ CPEB
ㅁ CRA
$\square$ IES
$\square$
$\square$ IESO
$\square$ COL

$\mathrm{N}^{\mathrm{e}}$ total de alumnado en el centro:

ㅁ menos de 50

ㅁ entre 50 y 100

믈 101 y 250

ㅁ entre 251 y 400

ㅁ entre 401 y 600

믈 601 y 800

ㅁ entre 801 y 1000

más de 1000

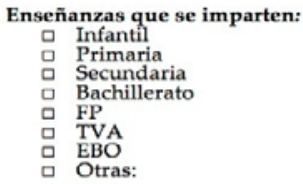

$\mathrm{N}^{\mathrm{Q}}$ total de alumnado:

$\mathrm{N}^{\circ}$ de alumnado con necesidades educativas especiales

$\mathrm{N}^{\bullet}$ de alumnado con necesidades específicas de apoyo educativo
EP: ESO:
EP: ESO:
EP: ESO:

Recursos personales del servicio de orientación en el centro educativo:

Si el centro no cuenta con el recurso, marcar en el valor 0 , si cuentan con medias jornadas, utilizar los valores .5.

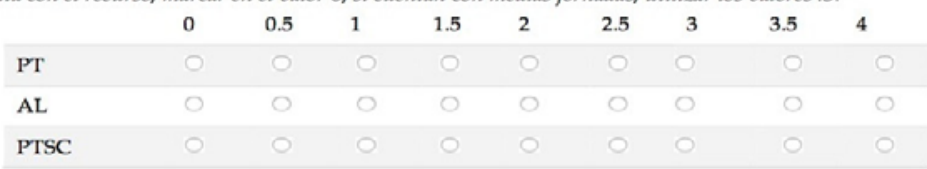

El centro participa en programas institucionales: SI NO

En el centro se organizan grupos de trabajo, cursos, La formación que se realiza en el centro, zestá relacionada con la atención a la diversidad?

SI NO

proyectos de formación... SI NO 


\section{BLOQUE IV. ESCALA LIKERT}

Basándote en la realidad educativa de tu centro y respecto a la Etapa, indica el grado de acuerdo respecto a las siguientes afirmaciones. Valora los ítems teniendo en cuenta que: 1= Muy en desacuerdo al 5= Muy de acuerdo

1. El profesorado conoce la normativa sobre atención a la diversidad.

2. La CCP funciona como elemento de coordinación y dinamización de las medidas que se adoptan para la atención a la diversidad del alumnado.

3. La organización del PAT contempla medidas que favorecen una atención personalizada del alumnado.

4. El Equipo Directivo se implica en la gestión de la atención a la diversidad.

5. El PEC recoge acuerdos de centro sobre atención a la diversidad ajustada a las necesidades del contexto (social, familiar, cultural, étnico, lingüístico...).

6. La concreción curricular en el centro está diseñada teniendo en cuenta una oferta plural.

7. El agrupamiento que se realiza del alumnado es flexible.

8. El profesorado utiliza metodologías que permiten atender a los diferentes ritmos, estilos de aprendizaje, intereses... del alumnado.

9. Los Equipos Docentes establecen acuerdos sobre las medidas a adoptar con el alumnado que presenta dificultades.

10. El profesorado conoce los perfiles del alumnado que se puede incorporar a cada medida de atención a la diversidad.

11. El centro establece reuniones de coordinación entre el profesorado que trabaja con el alumnado de NEE para realizar la planificación y el seguimiento de las adaptaciones.

12. El desarrollo de programas de mejora, innovación educativa incluye actuaciones que repercuten en la mejora de la atención a la diversidad.

13. Las medidas de atención a la diversidad recogidas en la normativa nos permiten dar una respuesta ajustada a nuestro alumnado.

14. Los espacios de los que dispone el centro para atender a la diversidad son adecuados.

15. Las medidas de atención a la diversidad son objeto de evaluaciones periódicas.

16. El centro dispone de procedimientos que facilitan la toma de decisiones sobre las medidas de atención a la diversidad a adoptar.

17. Las Programaciones docentes especifican las medidas a adoptar para dar una respuesta educativa ajustada a todo el alumnado.

18. Los órganos de coordinación docente acuerdan medidas para prevenir el absentismo.

19. El profesorado tutor informa periódicamente a las familias sobre las dificultades de aprendizaje, medidas de atención a la diversidad... que se adoptan con sus hijos/as.

20. El centro cuenta con protocolos para registrar las medidas de atención a la diversidad que se han adoptado con el alumnado a lo largo de la Etapa.

21. La formación del profesorado es suficiente para dar una respuesta adaptada a la diversidad del alumnado.

22. Las medidas de atención a la diversidad facilitan que el alumnado alcance los objetivos y las competencias básicas de la Etapa.

23. Las medidas de atención a la diversidad que se adoptan en tu centro (AF, Apoyo

Ordinario...) favorecen que el alumnado alcance los objetivos del nivel que está cursando.

24. El profesorado solicita a la Unidad de Orientación asesoramiento metodológico que les permita atender la diversidad en el aula.

25. El profesorado percibe el atender la diversidad del alumnado en el aula como un trabajo extra.

26. El desarrollo de actividades de sensibilización, talleres, etc. por parte de asociaciones, ONGDs,... contribuyen a generar una cultura de respeto a la diversidad en el centro.

27. Las medidas de atención a la diversidad favorecen la autoestima del alumnado.

28. Las actividades complementarias y extraescolares tienen en cuenta la diversidad del alumnado.

29. El equipo directivo garantiza los aspectos prescriptivos en materia de atención a la diversidad.

30. En la distribución del alumnado en grupos prevalece el criterio organizativo sobre el pedagógico.

31. El profesorado plantea las mismas actividades para todo el alumnado.

32. Los equipos docentes desarrollan las medidas que se deben de adoptar para dar una respuesta individualizada al alumnado. 
33. El profesorado conoce los criterios para la incorporación del alumnado a las distintas medidas de atención a la diversidad.

34. La evaluación de las medidas de atención a la diversidad es sistemática.

35. El número de reuniones que el profesorado mantiene con las familias del alumnado que presenta dificultades,.... es adecuado.

36. En el centro existe la posibilidad de realizar agrupamientos o fórmulas organizativas distintas a las ordinarias.

37. Cuando las medidas de atención a la diversidad que se aplican a un alumno/a no resultan eficaces, se adoptan las modificaciones oportunas.

38. El profesorado tiene en cuenta la diversidad del alumnado a la hora de tomar decisiones sobre los materiales que utiliza en el aula.

39. El agrupamiento del alumnado respeta criterios de heterogeneidad.

40. El profesorado del centro participa en acciones formativas (grupos de trabajo, cursos, seminarios...) para actualizar conocimientos relativos a la atención a la diversidad.

41. Las medidas de atención a la diversidad contribuyen a que el alumnado desarrolle al máximo sus capacidades, potencialidades...

42. Las familias participan en el seguimiento de las medidas de atención a la diversidad que se adoptan con sus hijos/as.

43. El profesorado considera la mejora del alumnado respecto a la actitud de trabajo, asistencia... como criterios que se han de tener en cuenta al valorar la eficiencia de las medidas de atención a la diversidad.

44. El profesorado con alumnado con NEE dispone de horario para coordinarse con los/as especialistas.

45. La organización de los recursos personales y materiales del centro se organizan con la finalidad de atender a la diversidad del alumnado.

Nota: En el IDEC-O B se duplica la escala de 45 items. Responden una para EP y otra para ESO.

\section{BLOQUE V. PREGUNTAS ABIERTAS}

Partiendo de la realidad de tu centro, señala aquellos aspectos que a tu juicio:

Favorecen la respuesta a la diversidad:

Dificultan la respuesta a la diversidad:

¿Qué propuestas de mejora realizarias? 